\title{
The burden of imported malaria in Cape Town, South Africa
}

\author{
J Opie, MB ChB, MRCP (UK), FRCPA (Haem); R Freeks, MB ChB; L A du Pisani, MB ChB, FCPath (SA) (Haem) \\ Division of Haematology, Faculty of Health Sciences, University of Cape Town and C17 Clinical Pathology Laboratory, National Health Laboratory \\ Service and Groote Schuur Hospital, Cape Town, South Africa
}

Corresponding author: J Opie (jessica.opie@uct.ac.za)

\begin{abstract}
Background. The Western Cape Province of South Africa (SA) is not malaria endemic; however, a considerable number of patients present with malaria to our healthcare services.

Objectives. To establish the frequency of patients presenting with malaria at Groote Schuur Hospital (GSH), Cape Town, SA, and to describe their demographics, clinical outcomes and laboratory findings.

Methods. An observational, retrospective, descriptive study was conducted, which included all patients presenting with smear-positive malaria to GSH over a 4-year period between 1 April 2008 and 31 March 2012.

Results. During the study period, 134 malaria patients presented to GSH for management; $85 \%$ ( $n=114$ ) were male, median age was 27 years. Of the total smear-positive tests, $96 \%(n=128)$ were Plasmodium falciparum, $3 \%(n=4)$ P. ovale, and in $1 \%(n=2)$ the species was not identified. The number of malaria patients increased markedly, from 6 cases in 2008 to 50 cases in 2012. Of the patients, $48.3 \%$ $(n=57)$ were from Somalia, 8.5\% $(n=10)$ from SA and 29\% $(n=30)$ from other African countries. One SA patient acquired transfusiontransmitted malaria from a pooled platelet product, and the other SA patients had travelled to malaria-endemic areas. The remaining cases were from countries outside of Africa, including 13\% $(n=15)$ from Bangladesh. Almost two-thirds $(62 \% ; n=72)$ were admitted to hospital with a median length of stay of 3 days (range 1 - 32). Clinical outcomes were good with only one death and the remaining patients being discharged.

Conclusion. Imported malaria is imposing a significant burden on health resources. The costs of medical care for the emergency treatment of foreign nationals needs to be recognised, and adequately budgeted for.
\end{abstract}

S Afr Med J 2014;104(5):347-349. DOI:10.7196/SAMJ.7904

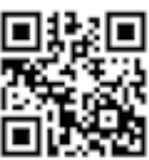

Only a small area within South Africa (SA) is malaria endemic and this is in the north-eastern part of KwaZulu-Natal, and in Mpumalanga and Limpopo provinces. ${ }^{[1]}$ All cases of malaria in other areas of SA are imported, either from malariaendemic parts of SA or from further afield.

A large number of migrants travel to SA for economic, political and social reasons. According to the United Nations Refugee Agency, SA was the leading worldwide destination country of new asylum seekers between 2006 and 2011. Between 2008 and 2012, the SA Department of Home Affairs registered 778600 new asylum applications, with Zimbabweans accounting for more than half of these. ${ }^{[2]}$ However, as only a proportion of migrants to SA are registered as asylum seekers, the true number of migrants is unknown.

Many foreign migrants come from malaria-endemic areas and present to SA healthcare services after arrival. Groote Schuur Hospital (GSH) in Cape Town is a tertiary hospital serving the Western Cape Province, with 975 beds and a busy medical casualty department that handles $\sim 40000$ patients/year.

The aim of this study was to establish the number of malaria patients presenting to GSH over the 4-year period between 1 April 2008 and 31 March 2012, and to describe their demographics, clinical outcomes and laboratory findings.

\section{Methods}

Using the laboratory information system at the National Health Laboratory Service (NHLS) at the GSH haematology laboratory, a database was compiled of all malaria tests performed during the 4-year study period. A malaria test includes a thick and thin malaria smear (using a Giemsa stain) and an immunochromatographic (ICT) malaria antigen test (BinaxNOW, Alere, USA). However, for our study, only the first smear-positive result was included for analysis as this is the gold standard. ${ }^{[3]}$ The ICT results were therefore not included. Repeat tests for the same patient were also excluded, unless performed $>1$ month after the initial test, in which case it was regarded as a second event. Only patients with smear-positive malaria who presented to GSH were included for analysis. Using results derived from the laboratory information system, laboratory findings were documented and patient medical records were then reviewed for data on patient demographics and clinical outcomes. The study was approved by the Faculty of Health Sciences Ethics Committee, University of Cape Town.

\section{Results}

During the 4-year study period, 1410 malaria tests were performed at the NHLS GSH haematology laboratory; 333 tests were smearpositive and 1077 -negative. Since GSH NHLS is a referral laboratory serving a wide region, including clinics and regional hospitals, only 134 of the total positive tests involved patients attending GSH. Of these, $85 \%(n=114)$ were male. The median age of patients was 27 years (range 2 - 59). Plasmodium falciparum was identified on the smear in $96 \%(n=128)$ of cases, $P$. ovale in $3 \%(n=4)$ of cases, and in two cases identification of the Plasmodium sp. was inconclusive due to a very low parasitaemia with only a single ring form identified. The median haemoglobin concentration on presentation was $11.9 \mathrm{~g} / \mathrm{dl}$ (range 1.3 - 17.7), the median platelet count was $75 \times 10^{\circ} / 1$ (range 13 
- 275), and the median total white cell count was $6.06 \times 10^{9} / 1$ (range 1.4 - 34) (Table 1$)$.

A total of 118 medical folders were retrieved; $48.3 \%(n=57)$ of patients originated from Somalia, $8.5 \%(n=10)$ from SA, $29 \%$ $(n=30)$ from other countries in Africa, and $15.5 \% \quad(n=18)$ came from countries outside Africa - mostly Bangladesh $(n=15$; $13 \%$ ) (Fig. 1). Of the SA patients, all had travelled to malaria-endemic areas apart from one patient who acquired malaria via a transfusion of platelet concentrate received while an inpatient at GSH. During the first year of study, six patients with malaria presented to GSH. This increased sharply to 24 cases in the second year and 54 and 50 cases in the third and fourth years, respectively (Fig. 2). Of the total, 38.5\%

Table 1. Haematology and parasitology results

\begin{tabular}{ll}
\hline $\begin{array}{l}\text { On presentation, median (range) } \\
\text { Haemoglobin }(\mathrm{g} / \mathrm{dl})\end{array}$ & $11.9(1.3-17.7)$ \\
$\quad$ Platelet count $\left(\times 10^{9} / \mathrm{l}\right)$ & $75(13-275)$ \\
$\quad$ White cell count $\left(\times 10^{9} / 1\right)$ & $6.06(1.4-34)$ \\
Species identified by microscopy, $n(\%)(N=134)$ & $128(96)$ \\
$\quad$ Plasmodium falciparum & $4(3)$ \\
$\quad$ Plasmodium ovale & $2(1)$ \\
$\quad$ Inconclusive & \\
*In each of these cases a single ring form was seen; therefore the parasitaemia was too low to conclusively identify the \\
Plasmodium sp.
\end{tabular}

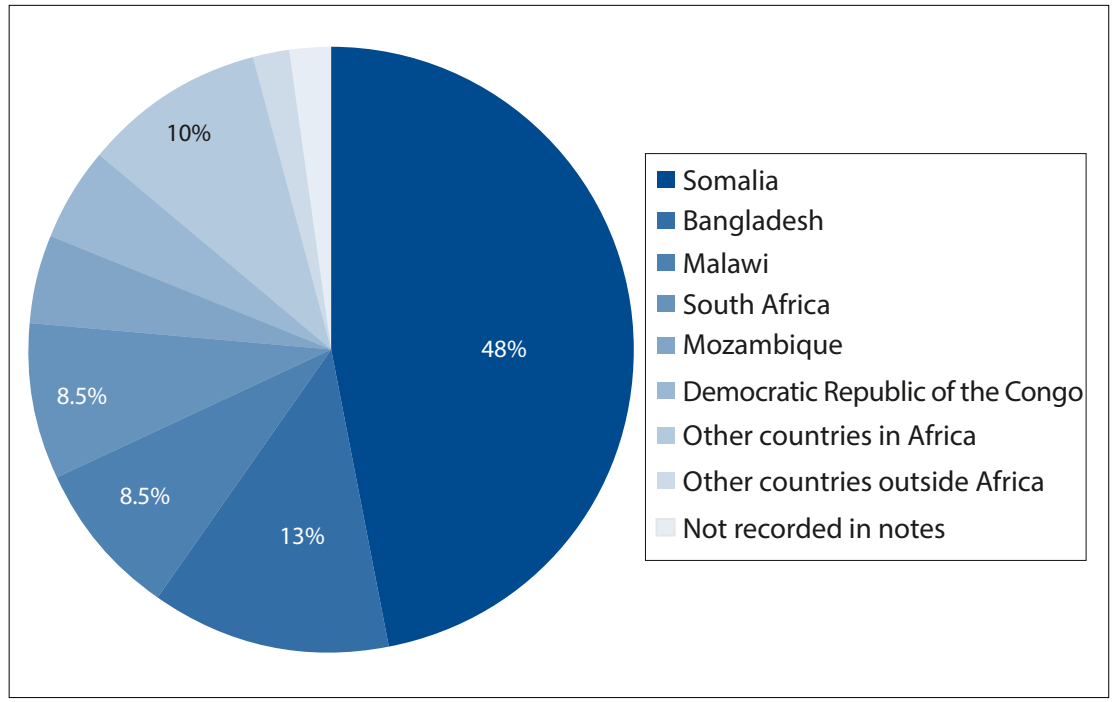

Fig 1. Distribution of malaria patients by country of origin.

Table 2. Demographics and clinical outcomes

\begin{tabular}{ll}
\hline Malaria patients (male), \% $(N=134)$ & 85 \\
Age (years), median (range) & $27(2-59)$ \\
Clinical outcomes, $n(\%)^{*}$ & $72(62)$ \\
Admitted to ward & $44(38)$ \\
Liver dysfunction & $13(11)$ \\
Renal dysfunction & $6(5)$ \\
Cerebral malaria & $45(38)$ \\
Outpatient management & $1(<1)$ \\
Deaths & $3(1-32)$ \\
Duration of admission (days), median (range) & \\
${ }^{*}$ A total of 118 medical folders were retrieved. One patient refused admission. &
\end{tabular}

$(n=45)$ were managed as outpatients and $62 \%(n=72)$ were admitted to hospital wards. One patient refused admission. One death occurred in a patient with $P$. falciparum complicated by cerebral malaria. The remainder were discharged. The median hospital stay was 3 days (range 1 - 32). Of the total patients for whom folders were found, $38 \%(n=44)$ had liver dysfunction, $11 \%$ $(n=13)$ had renal dysfunction, and 5\% $(n=6)$ had documented cerebral malaria (Table 2).

\section{Discussion}

Our study findings demonstrate a marked increase in patients presenting with malaria to $\mathrm{GSH}$ during the 4-year study period between 2008 and 2012. This is in contrast to malaria-endemic regions of SA where cases of malaria are showing a downward trend. ${ }^{[1]}$ Of the study patients, $91.5 \%$ came from countries outside SA, which is in keeping with the pattern of increasing migration into this country and into the Western Cape Province. The findings are similar to those of a recent study on children with sickle cell disease presenting to Red Cross War Memorial Children's Hospital, Cape Town, which demonstrated an increase in new patients over a 10-year period and found that 93\% of the patients originated from African countries outside SA. ${ }^{[4]}$ These findings should therefore be regarded as an indicator of the increased migrant population presenting to SA healthcare services across all fields of medicine.

The commonest country of origin of malaria patients in our study group was Somalia ( $48 \%$ of patients). A significant proportion came from Bangladesh (13\%), having travelled via other countries in Africa. Two-thirds of patients required hospital admission, the mean length of admission being 3 days. One SA patient acquired transfusion-transmitted malaria from a pooled platelet product. Transfusiontransmitted malaria is an exceedingly rare occurrence. Since 1985, the Western Province Blood Transfusion Service has confirmed three cases of transfusion-associated malaria, two from red cell concentrates and one from pooled platelets. The transfusion donors of these three cases were found to have very low levels of parasitaemia, to have previously lived in malaria-endemic areas and to be clinically asymptomatic.

The clinical outcomes in this study group were good with only one death $(<1 \%$ of all patients). This low mortality is a likely reflection of the semi-immunity of the majority of our patients who came from malaria-endemic areas. ${ }^{[5]}$ This is in contrast 


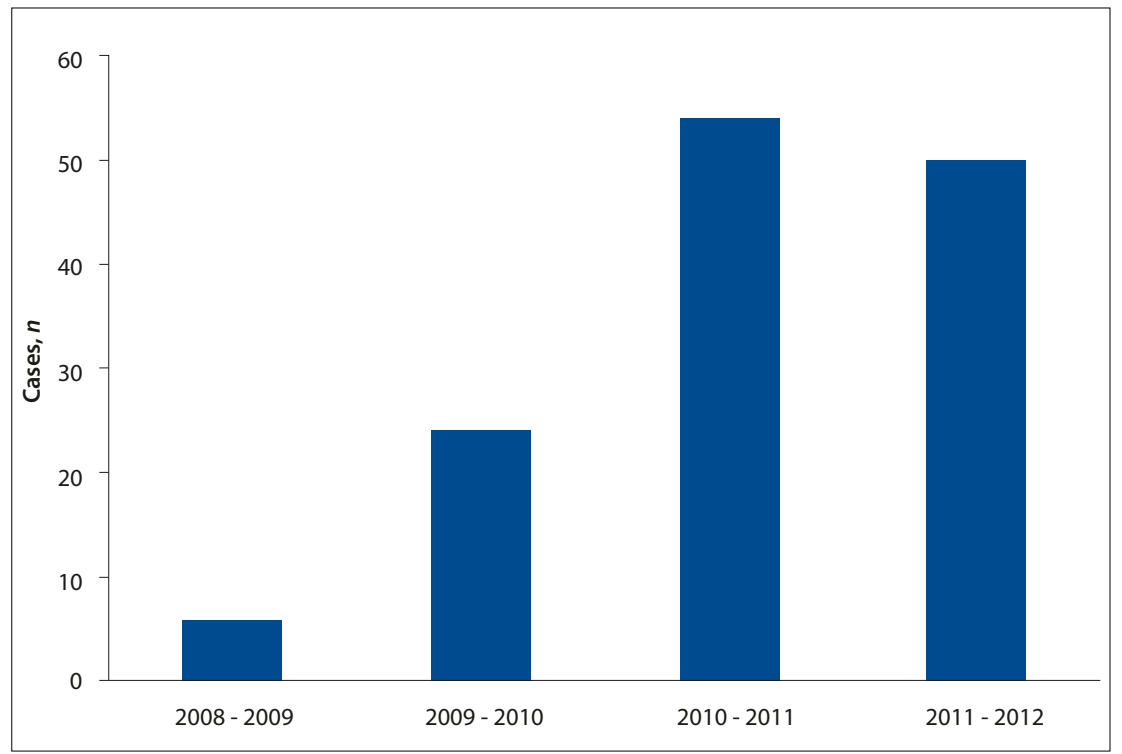

Fig. 2. Frequency of malaria at Groote Schuur Hospital over the 4-year study period (N=134).

to non-malaria immune patients who are known to have a higher mortality if contracting malaria following travel to an endemic area. ${ }^{[6]}$

Rapid testing for malaria species using ICT rapid diagnostic tests that detect parasite antigen using a dipstick format are now widely available. ICT tests are particularly useful in remote areas where microscopy is unavailable or where laboratory personnel are inexperienced in the interpretation of smear findings. ICT tests are also quick and easy to perform at the point of care, however, they have drawbacks particularly in terms of sensitivity. The BinaxNOW ICT test used at GSH NHLS is known to have a high sensitivity for the diagnosis of $P$. falciparum, but is less sensitive for non-P. falciparum malaria. ${ }^{[7]}$ Thin and thick smear blood film preparations therefore remain the 'gold standard' for malaria diagnosis and a first thin and/or thick smear-positive test was used to determine inclusion in this study.
The tertiary healthcare services in Cape Town are seeing increased numbers of patients with imported malaria who have originated from countries outside SA.
This may be regarded as an indicator of the changing demographics of patients presenting to the SA healthcare services, including to the general medical, surgical and obstetric sectors. This growing trend is imposing a significant burden on healthcare services, which are already under strain. This issue needs to be addressed in a constructive manner. We recommend that a formal budget allocation be made by the Department of Health for emergency healthcare of foreign migrants.

Acknowledgement. The authors thank Dr Gregory Bellairs and the staff of the Western Province Blood Transfusion Service who investigated the case of transfusion-transmitted malaria.

\section{References}

1. Maharaj R, Raman J, Morris N, et al. Epidemiology of malaria in South Africa: From control to elimination. S Afr Med J 2013;103(10 Suppl 2):779-783 [http://dx.doi.org/10.7196/SAMJ.7441]

2. United Nations High Commissioner for Refugees. United Nations High Commissioner for Refugees.
Displacement: The New 21st Century Challenge - UNHCR Displacement: The New 21st Century Challenge - UNHCR
Global Trends 2012. Geneva: UNHCR, 2012. http://unhcr.org/ globaltrendsjune2013/ (accessed 5 March 2014).

3. Frean J, Poonsamy B, Shandukani B, Moonasar D, Raman J. Case management of malaria: Diagnosis. S Afr Med J 2013;103(10 Suppl 2):789-793 [http://dx.doi.org/10.7196/SAMJ.7442]

4. Wonkam A, Ponde C, Nicholson N, Fieggen K, Ramessar R, Davidson A. The burden of sickle cell disease in Cape Town. S Afr Med J 2012;102(9):752-754 [http://dx.doi.org/10.7196/ SAMJ.5886]

5. Pistone T, Diallo A, Mechain M, Receveur M-C, Malvy D, Epidemiology of imported malaria give support to the hypothesis of 'long-term' semi-immunity to malaria in sub-Saharan African of 'long-term' semi-immunity to malaria in sub-Saharan African
migrants living in France. Travel Med Infect Dis 2014;12(1):48migrants living in France. Travel Med Infect Dis 2014

6. Checkley A, Smith A, Smith V, et al. Risk factors for mortality Checkley A, Smith A, Smith V, et al. Risk factors for mortality
from imported falciparum malaria in the United Kingdom over from imported falciparum malaria in the United Kingdom over
20 years: An observational study. BMJ 2012;344:e2116. [http:// dx.doi.org/10.1136/bmj.e2116]

7. Farcas G, Zhong K, Lovegrove F, et al. Evaluation of the Binax NOW ICT test versus polymerase chain reaction and microscopy for the detection of malaria in returned travellers. Am J Trop Med Hyg 2003;69(6):589-592.

8. World Health Organisation. World Malaria Report 2012. Geneva: WHO, 2012:59. http://www.who.int/malaria/ publications/world_malaria_report_2012/en/index.html (accessed 18 December 2013).

9. Kelton J, Keystone I- Moore J et al. Immune-mediated thrombocytopenia of malaria. J Clin Invest 1983;71(4):832-836. 